\title{
Letter in Reply
}

\section{Reusable blood collection tube holders are implicated in nosocomial hepatitis $C$ virus transmission}

\author{
Vincent C. C. Cheng MD ${ }^{1,2}$, Shuk-Ching Wong MNurs ${ }^{2}$, Sally C. Y. Wong FRCPath ${ }^{1}$, Siddharth Sridhar FRCPath ${ }^{3}$, \\ Cyril C. Y. Yip PhD ${ }^{1}$, Jonathan H. K. Chen $\mathrm{PhD}^{1}$, James Fung MD ${ }^{4}$, Kelvin H. Y. Chiu MRCP ${ }^{1}$, Pak-Leung Ho MD ${ }^{3}$, \\ Sirong Chen $\mathrm{PhD}^{5}$, Ben W. C. Cheng MHKCRRT(CNMR) ${ }^{5}$, Chi-Lai Ho MD ${ }^{5}$, Chung-Mau Lo $\mathrm{MS}^{6}$ \\ and Kwok-Yung Yuen $\mathrm{MD}^{3}$ \\ ${ }^{1}$ Department of Microbiology, Queen Mary Hospital, Hong Kong Special Administrative Region, China, ${ }^{2}$ Infection Control Team, Queen Mary Hospital, Hong \\ Kong West Cluster, Hong Kong Special Administrative Region, China, ${ }^{3}$ Department of Microbiology, Li Ka Shing Faculty of Medicine, The University of Hong \\ Kong, Hong Kong Special Administrative Region, China, ${ }^{4}$ Department of Medicine, Queen Mary Hospital, Hong Kong Special Administrative Region, China, \\ ${ }^{5}$ Department of Nuclear Medicine \& Positron Emission Tomography, Hong Kong Sanatorium and Hospital, Hong Kong Special Administrative Region, China and \\ ${ }^{6}$ Department of Surgery, Li Ka Shing Faculty of Medicine, The University of Hong Kong, Hong Kong Special Administrative Region, China
}

To the Editor-In response to the letter by Tsang et al, ${ }^{1}$ we offer the following point-by-point rebuttal. Almost all hospital outbreak investigations require direct observation of patient care practices that are confounded by the Hawthorne effect, leading to the underestimation of incorrect practices. Despite this limitation, our direct observation showed that our phlebotomists had not been trained to comply with 2 manufacturers' instructions: (1) the need for disinfection of reusable blood collection tube holders (RBCTH) between every patient and (2) the release of tourniquet immediately after blood starts flowing into the specimen tube to minimize backflow. ${ }^{2-4}$ These noncompliant practices had been adopted by all phlebotomists since the introduction of RBCTH into Hong Kong public hospitals.

The male source patient and the female victim were housed in the same ward served by 1 or more phlebotomists. Our computerized barcoding system recorded 34 phlebotomists with 54 visits to this ward for all patients between August 6, 2017, and August 19, 2017. The same phlebotomist collected blood from the source patient before collecting blood from the female victim in the morning shift on August 9, 2017, (phlebotomist A) and on August 11, 2017 (phlebotomist B). Of 29 phlebotomists being interviewed, 28 reported the sole use of RBCTH kept in the ward's phlebotomy trolley where the HCV-positive RBCTH was found, including those who provided services on August 9, 2017, and August 11, 2017. Because HCV remains infectious for 6 weeks in the environment, patients were at risk of exposure whether the same or different phlebotomists took blood from the victim before or after the source patient, as long as the HCVcontaminated RBCTH in this phlebotomy trolley was in use. Using this barcoding system, we reviewed an earlier case of nosocomially acquired HCV in a 94-year-old female (supplementary material online). A phlebotomist took blood from a

\footnotetext{
Author for correspondence: Kwok-Yung Yuen, Carol Yu Centre for Infection, Department of Microbiology, Li Ka Shing Faculty of Medicine, The University of Hong Kong, Hong Kong Special Administrative Region, China. E-mail: kyyuen@hku.hk

Cite this article: Cheng VCC, et al. (2019). Reusable blood collection tube holders are implicated in nosocomial hepatitis C virus transmission. Infection Control \& Hospital Epidemiology 2019, 40, 252-253. doi: 10.1017/ice.2018.314
}

78-year-old female HCV-positive patient on April 7, 2016, and collected blood from this victim immediately afterward. Again, the RBCTH could have been the vector; extensive investigation did not identify any other modes of transmission.

In addition to the RBCTH, only 1 glucometer and 1 phlebotomy trolley in use could be sampled for HCV on December 5, 2017. Our direct observation showed no practice irregularities in venous catheter insertion or multiple-dose drug-vial sharing. The presence of $\mathrm{HCV}$ inside the RBCTH with high degree of sequence similarity to source patient and victim $\mathrm{HCV}$ isolates clearly demonstrated that the phlebotomists had used this RBCTH and that blood contamination inside this RBCTH had occurred. Combination with the information from our barcoding system, the possibility of both source and victim sharing this RBCTH has to be entertained.

Until now, none of the reported HCV hospital outbreaks have utilized whole-virus genome sequencing, although partial-genome quasi-species sequencing was advocated by Campo et al. ${ }^{5}$ Most previous reports used the hypervariable region (E1 and E2 HVR), with only $140 \mathrm{bp}$ to $411 \mathrm{bp}$ sequenced. ${ }^{6,7}$ Others have used the NS5b, with 328 bp sequenced. ${ }^{8}$ Only 1 study has evaluated environmental samples comparing 81 bp of HVR between patients and environmental samples. In this case-control study suggesting the sharing of multiple-dose heparin vial as the source, no HCV could be detected in these vials. ${ }^{9}$ Another case-control study using multivariate analysis showed that international normalized ratio (INR) monitoring by phlebotomy and podiatry were risk factors for HCV acquisition, ${ }^{10}$ and next-generation sequencing was used to analyze $291 \mathrm{bp}$ of HVR quasi-species instead of the entire genome. In this study, $<3 \%$ ( $9 \mathrm{bp}$ ) of the genome was used as evidence of clonality between HCV strains. ${ }^{10}$ Thus, none of the phylogenetic studies of HCV outbreak were as stringent as ours. With only 3 nucleotide positions of divergence of $653 \mathrm{bp}$ (HVR) between the HCV sequences from the RBCTH, the source patient, and the victim, we thus confirmed clonality.

As for the phlebotomy simulation experiments, we used only $\mathrm{HCV}$-positive plasma because no HCV-positive archived EDTA blood was available. Blood is denser and more adhesive than plasma, which would lead to a larger volume of inoculum remaining on 
contaminated surfaces. The dipping of $5 \mathrm{~mm}$ of the tip of a rubber sleeve picked up $0.06 \mu \mathrm{L}($ mean $\pm \mathrm{SD}, 0.02 \mu \mathrm{L})$ of the $\mathrm{HCV}$-positive plasma. This volume was so low that this inoculum was invisible to the naked eye and was less than the $1.4 \mu \mathrm{L}$ (mean volume) inoculum from a hollow-needle needlestick injury. ${ }^{11}$ Therefore, this procedure was a reasonable substitute for in vitro simulation.

The objective of the 2 radionuclide experiments was to dynamically investigate the possibility of reflux communication from $\mathrm{RBCTH}$ into the patient during a simulated phlebotomy (not virus transmission). A reflux can only happen in 2 conditions: bidirectional patency and pressure difference. Blood has both solution and colloidal properties; therefore, it is a homogeneous carrier medium for transport of all its normal constituents (from cells to molecules). Ideally, 99mTc-labeled blood (eg, used for study of gastrointestinal bleeding) could be used for this experiment. In addition, a 16G-20G needle has an inner cross-sectional area 7,000-27,000 times that of red blood cells, which is thus statistically and obviously not a limiting factor for the size of a molecule or virus. The pressure change created by releasing the manual pressure from the saline bag was unquantified (please note that we did not say negative pressure). However, during phlebotomy, differential pressure changes are known to be operator and patient dependent; therefore, they vary and, likewise, were unquantified. Our experiment clearly demonstrated that even a very gentle manually applied dynamic pressure difference on the saline bag could induce a patent route of reflux from the needle side to the bag side during the simulation of phlebotomy with RBCTH.

Regarding the backflow of blood, the crux of the matter is the sudden release of the tourniquet, which allows the venous blood under positive pressure below the tourniquet (at antecubital fossa level) to go above the tourniquet at arm level. The HCVcontaminated "blood pool" between sleeved-needle and the sleeve then flows back into the patient. Thus, creating negative pressure in the venous system is unnecessary for causing the backflow. As long as there is a pressure gradient between the vein below and above the tourniquet, the HCV-contaminated blood can flow into the patient when the tourniquet is released.

We did not find any peer-reviewed journal, publication-quality methodology or data in reference 5 cited by Tsang et al. This citation refers to the evaluation of a single-use tube holder and the low risk of backflow from the vacuum specimen container through the sleeved needle back into the patient, whereas our study refers to $\mathrm{RBCTH}$ and the risk of backflow from the blood pool between the sleeve and the sleeve needle (not the vacuum specimen tube) into the patient. Both our testing with $\mathrm{HCV}$-positive plasma (with virions much bigger than technetium) and radioactive technetium (visible on radiation scanning) showed significant backflow into the patient's side, therefore posing a risk to the patient. Most importantly, our phlebotomists have not been trained to comply with the manufacturer's instruction of the need to release the tourniquet once the blood starts to flow into the vacuum specimen tube.

Without providing evidence that the blood inside the tube holder belonged to the patient's own blood and not the source patient, the opinion of Tsang et al is speculative. We showed that the genetic sequences from the source, the $\mathrm{RBCTH}$, and the victim were $99.54 \%$ identical.

We believe that the evidence presented in our original article and our present rebuttals are scientifically well grounded, contrary to the description by Tsang et al as "exaggerated, flawed, superfluous, hasty, premature or disproportionate." Our data were scrutinized by the Hospital Authority governing all public hospitals in Hong Kong. The Hospital Authority terminated the further use of RBCTH to protect patients. We now follow the best practice in the United States, United Kingdom, and Australia of using only disposable single-use tube holders. If RBCTH are ever used, the phlebotomist must comply with the manufacturers' instructions by disinfecting all RBCTH between patients and by releasing the tourniquet once blood starts to flow into the specimen containers. Unfortunately, these important instructions have not been provided to frontline healthcare workers for many years; thus, more cases presenting with $\mathrm{HCV}$ cirrhosis and hepatocellular carcinoma may be expected over time.

\section{Acknowledgments. None.}

Financial support. This work was supported in part by the donations of $\mathrm{Mr}$. Michael Tong, Providence Foundation (in memory of the late LuiHac Minh) and the Hong Kong Hainan Commercial Association and by funding from the Consultancy Service for Enhancing Laboratory Surveillance of Emerging Infectious Diseases of the Department of Health, Hong Kong Special Administrative Region, and the Collaborative Innovation Center for Diagnosis and Treatment of Infectious Diseases, the Ministry of Education of China.

Conflicts of interest. All authors declared no potential conflicts of interest.

Supplementary material. To view supplementary material for this article, please visit https://doi.org/10.1017/ice.2018.314

\section{References}

1. Tsang DNC, Ip M, Chan PKS, et al. Are reusable blood collection tube holders the culprit for nosocomial hepatitis $\mathrm{C}$ virus transmission? Infect Control Hosp Epidemiol 2018;X:X-X.

2. BD vacutainer eclipse blood collection needle. Becton Dickinson website. https://www.bd.com/documents/bd-legacy/quick-guide/blood-and-urinecollection/PAS_BC-BD-Eclipse-Blood-Collection-Needle-Points-to-Practice_ QG_EN.pdf. Accessed October 23, 2018.

3. VACUETTE blood collection tubes. Evacuated blood collection system for in vitro diagnostic use. Greiner Bio-One website. https://www.gbo.com/ fileadmin/user_upload/Downloads/IFU_Instructions_for_Use/IFU_ Instructions_for_Use_Preanalytics/English/980200_EN_rev20.PDF. Accessed October 23, 2018.

4. VACUETTE blood collection techniques. Greiner Bio-One website. https://www.gbo.com/fileadmin/user_upload/Downloads/Brochures/ Brochures_Preanalytics/English/980063_BloodCollectionTechniques_e_rev07_0116_lowres.pdf. Accessed October 23, 2018.

5. Campo DS, Xia GL, Dimitrova Z, et al. Accurate genetic detection of hepatitis $\mathrm{C}$ virus transmissions in outbreak settings. I Infect Dis 2016;213:957-965.

6. Widell A, Christensson B, Wiebe T, et al. Epidemiologic and molecular investigation of outbreaks of hepatitis $\mathrm{C}$ virus infection on a pediatric oncology service. Ann Intern Med 1999;130:130-134.

7. Grethe S, Gemsa F, Monazahian M, Böhme I, Uy A, Thomssen R. Molecular epidemiology of an outbreak of HCV in a hemodialysis unit: direct sequencing of HCV-HVR1 as an appropriate tool for phylogenetic analysis. J Med Virol 2000;60:152-158.

8. Garvey MI, Bradley CW, Holden KL, et al. Use of genome sequencing to identify hepatitis $\mathrm{C}$ virus transmission in a renal healthcare setting. J Hosp Infect 2017;96:157-162.

9. Girou E, Chevaliez S, Challine D, et al. Determinant roles of environmental contamination and noncompliance with standard precautions in the risk of hepatitis $\mathrm{C}$ virus transmission in a hemodialysis unit. Clin Infect Dis 2008;47:627-633.

10. Calles DL, Collier MG, Khudyakov Y, et al. North Dakota Hepatitis C Virus Investigation Team. Hepatitis $C$ virus transmission in a skilled nursing facility, North Dakota, 2013. Am J Infect Control 2017;45:126-132.

11. Napoli VM, McGowan JE Jr. How much blood is in a needlestick? J Infect Dis $1987 ; 155: 828$. 